\title{
Name index
}

Agis, E. 68

Ahmed, S. 145, 165

Akyüz, Y. 132-134, 146

Alesina, A. 22, 23

Allain, O. 49, 160

Allami, C. 59

Amadeo, E. 42

Amiti, M. 91

Ampudia Márquez, N.A. 93

Arestis, P. 5, 93, 140

Arrow, K. 76-77

Arslanalp, S. 132

Asimakopulos, A. 27, 32

Aspromourgos, T. 30

Assous, M. 15, 30

Athukorala, P.C. 164

Atkinson, A. 6, 7

Auerbach, P. 39

Avdjiev, S. 132-133, 165

Azpiazu, D. 73

Backhouse, R.E. 1

Backus, J. 75

Baran, P. 35

Barba, A. 4

Barnes, M. 93

Basualdo, E. 57-59

Baumann, B. 165

Bedirhanoglu, P. 137

Belabed, C. 80

Bellamy Foster, J. 20, 35

Benigno, G. 130, 147, 162

Bentolila, S. 7

Berg, A. 54

Bernanke, B. 1

Beveridge, W. 24, 30

Bezemer, D. 83

Bhaduri, A. 31-37, 40, 51, 72, 131, 143

Bibow, J. 164

Bindseil, U. 100

Blecker, R. 3, 33, 38, 49, 72, 131, 144
Boianovsky, M. 1, 30

Borio, C. 100

Borrill, P. 83

Bortz, P. 118

Botta, A. 162

Bowles, S. 55

Boyer, R. 55

Brainard, W. 75

Brown, M. 146

Bruno, V. 145

Buiter, W. 75

Burgess, S. 9

Burkett, P. 131

Bussière, M. 164

Butzen, P. 134, 145

Caiani, A. 78, 79, 83

Calcagno, A.F. 135

Calvo, G. 144

Canitrot, A. 58

Carrión-Alvarez, M. 78

Carter, S. 4

Carvalho, L. 47, 54

Caserta, M. 40

Cassetti, M. 48, 49, 140

Caverzasi, E. 75, 77, 79

Cecchetti, S. 8-9

Chandrasekhar, C.P. 11

Chang, H.J. 136

Checchi, D. 7

Chick, V. 40

Chinn, M. 145

Chirinko, R. 106

Chui, M. 132, 133, 165

Chutasripanich, N. 98

Cibils, A. 59

Cincotti, S. 83

Cingano, F. 54

Cirera, X. 136

Claessens, S. 144

Clark, J.B. 21, 22 
Clement, P. 135

Converse, N. 130, 147, 162

Copeland, M. 75

Coremberg, A. 57, 60

Cornwall, J. 48

Cororaton, A. 93

Correa, E. 135

Cripps, F. 76

Crotty, J. 136, 137

Cutler, D.M. 98

Da Silva, C.E.S. 92

Dabla-Norris, E. 9, 54

Dafermos, Y. 79, 80

Daigle, G. 78, 81, 83, 98

Dalle, D. 103

Dallery, T. 42, 80, 140

Damill, M. 73

Davidson, P. 75

De Cecco, M. 129

De Grauwe, P. 98

De Lucchi, J.M. 136

De Vries, G. 130, 163

De Vries, K. 130, 163

Debreu, G. 76, 77

Del Monte, A. 32

Deroose, M. 134, 145

Diaz-Alejandro, C. 135

Disyatat, P. 100

Dobb, M. 21

Dornbusch, R. 129

Dos Santos, C.H. 75-77, 80

Downward, P. 73

Drehmann, M. 143

Dumenil, G. 32, 35, 39-40, 42, 43

Dünhaupt, P. 9

Dutt, A.K. 30, 31-32, 40, 49, 131, 140

Dymski, G. 137

Eatwell, J. 80

Ebeke, C. 109, 132

Ederer, S. 55

Edwards, S. 164

Ehnts, D. 78

Enders, W. 73

Engel, C. 146

Epstein, G. 8, 9, 79, 164

Fazzari, S. 143

Feiwel, G. 15
Feldman, E. 58

Felipe, J. 116-117

Fender, I. 132

Ferreres, O. 60

Fisher, I. 30

Fleming, M. 81, 106

Foley, D. 19

Fontana, O. 78, 80

Forbes, K.J. 144

Fornaro, L. 130, 147, 162

Frankel, J. 98

Friedman, M. 1, 93

Frondizi, A. 58

Froot, K. 98

Fry, M. 2

Furceri, D. 9, 130, 141, 147

Galanis, G. 54, 59-62, 157

Galbraith, J.K. 2

Gallagher, K. 130, 164, 165

Galor, O. 23, 54

García-Peñalosa, C. 7

Gasparini, L. 68

Gaulier, G. 116

Gerchunoff, P. 57

Ghosh, A. 129

Ghosh, S. 144

Giroud, X. 143

Godin, A. 75, 77-80, 162

Godley, W. 48, 75-83, 94, 96-97, 100-101

Goldberg, P.K. 5, 7

Gordon, D. 55

Gottschalk, R. 136

Grabel, I. 165

Graña, J. 58-60

Greene, W. 73

Greenspan, A. 7-8

Greiff, M.83

Griffith-Jones, S. 136, 164

Grimaldi, M. 98

Hagemann, H. 30

Haldane, A. 162

Harcourt, G.C. 73

Harrod, R.F. 26-27

Harvey, J. 98, 146

Hawtrey, R. 21

Hayek, F. 21, 22

Hein, E. 6, 8-9, 41, 44-48, 53-55, $60-66,80,91,114,143$ 
Helleiner, E. 10

Hicks, J.R. 7, 40

Hodgson, G. 78

Hubbard, R.G. 143

Humphrey, T. 118

Ide, S. 134, 145

Itskhoki, O. 91

Izurieta, A. 83

Jara, A. 165

Jaumotte, F. 7, 9, 130, 141, 147

Jayadev, A. 5, 9, 130, 147

Jevons, W. 30

Judd, K. 83

Juselius, K. 73

Juselius, M. 143

Kaldor, N. 17, 25, 27-29, 30, 32, 45, 48, $94,99,163$

Kalecki, M. 13, 15, 20, 27-28, 30, 31, $35,46,92,103$

Kaltenbrunner, A. 134, 145

Keifman, S. 73

Kennedy, D. 58-60

Keynes, J.M. 10, 13, 17, 19, 22-29, 30, $31,74,82,99,129$

Kharroubi, E. 8-9

Kim, J.H. 47, 78

King, J.E. 78

Kinsella, S. 78, 80, 83

Kirschenmann, K. 146

Kleinknecht, A. 94

Konings, J. 91

Korinek, A. 129

Kregel, J.A. 28, 111, 135-137, 159, 163

Kumar, U. 116-117

Kurz, H. 30, 35-37, 39, 40, 48

La Marca, M. 72, 131, 143-144

Lanata Briones, C. 59

Lavagna, R. 59

Lavoie, M. 3, 13, 31-32, 35-37, 39-48, 73, 75-83, 91, 94, 96-101, 106, $108,118,140,143,146$

Lawson, T. 78

Lazonick, W. 9

Le Heron, E. 79, 81

Lee, F. 73, 91

Leiderman, R. 144
León-Ledesma, M.A. 92

Lerner, A.P. 49, 103

Levy, D. 35, 39-40, 42-43

Lindenboim, J. 59

Llach, L. 57

Lo Vuolo, R. 59

López G., J. 15, 30, 55, 135, 157

López-Pérez, V. 93

Loungani, P. 9, 130, 141, 147

Lu, Y. 109, 132

Lucarelli, S. 78-79

Lucas Jr., R. 1, 93

Luxembourg, R. 20, 35

Lye, J. 93

Maes, I. 135

Mantey, C. 95, 160

Marcuzzo, M.C. 30

Marglin, S. 31-37, 40, 51, 143

Marshall, A. 49, 103

Martin, B. 81

Marx, K. 19-20, 74

Mazier, J. 82

Mazzucato, M. 163

McCarthy, J. 92

McCauley, R. 148

McCombie, J. 28, 72, 82, 94, 161

McDonald, I. 93

McGuire, P. 148

McKinnon, R. 2, 162

McLeay, M. 99-100

Mendieta-Muñoz, I. 93

Menger, K. 30

Michelena, G. 82

Milberg, W. 93

Mirowski, P. 30

Mirrlees, J. 27-28

Mises, L. 21

Missaglia, M. 49, 162

Mondino, T. 145

Moosa, I. 98

Mott, T. 40

Mouakil, T. 80

Mueller, H. 143

Mundell, R. 81, 106

Naastepad, C.W.M. 4-5, 48, 55, 93-94, $116,136,155,159$

Ndikumana, L. 143

Neftci, S.N. 137 
Nell, E.J. 83

Nersisyan, Y. 99

Nier, E. 145

Nikiforos, M. 43, 83, 117

Nogueira Junior, R. 92

Noland, M. 136

O'Shea, T. 78

O'Sullivan, M. 9

Ocampo, J.A. 164

Ochsen, C. 47

Olaberría, E. 165

Olivei, G. 93

Onaran, O. 49, 52-55, 59-62, 67, 157

Ongena, S. 146

Ono, F.H. 48

Oreiro, J.L. 48

Orhangazi, O. 9

Ostry, J. 54, 129

Pacioli, L. 75

Painceira, J.P. 134, 145

Palley, T. 1, 8, 48, 117

Palma, J.G. 134, 136

Palumbo, A. 40

Panigo, D. 59, 68

Papadimitriou, D. 79, 83, 117

Papatheodorou, C. 80

Pareto, V. 30

Park, C.H. 153

Park, H.J. 136

Pasinetti, L. 30

Passarella, M. 80

Patnaik, P. 164

Pavcnik, N. 5, 7

Peach, R. 93

Pérez Caldentey, E. 82

Perón, J.D. 57

Perón, M.E. 58

Perotti, R. 22

Petersen, B. 143

Philippon, T. 9

Phillips, A.C. 93-94, 117, 139

Pigou, A.C. 22

Piketty, T. 1-2, 6-7

Pilkington, M. 81

Pivetti, M. 4, 19, 39-40

Poterba, J.M. 98

Power, D. 9
Pugno, M. 28, 94

Pyatt, G. 75

Quayyum, S. 145

Quesnay, F. 74

Radia, A. 99-100

Rajan, R. 7, 23

Rajapatirana, S. 164

Ramírez-Gastón, P. 47

Reinhart, C. 99, 144

Rey, H. 130, 144-146

Reynolds, P. 73

Rezai, A. 47, 54

Ricardo, D. 17-21

Rich, R. 93

Robbins, L. 22

Roberto, M. 83

Robertson, D.H. 99, 130

Robinson, J. 25, 27-29, 32, 45, 140

Robinson, S. 75

Rochon, L.P. 48, 99, 140

Rodriguez, F. 5

Rodrik, D. 23

Rogoff, K. 99

Romer, P. 22

Rossi, P. 98

Round, J.I. 75

Rowthorn, R. 3, 31-32, 34, 40, 48, 94, 140

Ryoo, S. 81

Sadowski, Z. 30

Saez, E. 7

Saint-Paul, G. 7

Salvadori, N. 30

Sanchez, A. 157

Sasaki, H. 140

Sawyer, M. 30, 140

Schoder, C. 9, 43

Schroeder, E. 116

Schumpeter, J. 21, 30, 41, 74, 79, 99

Sedik, T.S. 145

Seguino, S. 159

Seppecher, P. 83

Serrano, F. 106, 160

Setterfield, M. 48, 140

Shaikh, A. 20, 35

Sharpe, S. 106

Shaw, E. 162 
Shin, H.S. 81, 132, 133, 145, 165

Skidelsky, R. 30

Skott, P. 35, 39-46, 81

Slattery, E. 40

Smith, A. 17, 30, 99

Solow, R. 22, 26

Sommer, J. 58

Soro, B. 28, 94

Spanos, A. 157

Spronk, R. 98

Sraffa, P. 36

Stehrer, R. 55

Steindl, J. 39

Stirati, A. 9, 18

Stock, J. 1, 93

Stockhammer, E. 4, 6-7, 9, 48-49, $52-53,55,130,147$

Storm, S. 4, 5, 48, 55, 93-94, 116, 136, 155,159

Studart, R. 163

Suarez, G. 106

Summa, R. 106

Summers, L. 1, 4, 98

Sushko, V. 132, 148

Sweezy, P. 19-20, 35

Szeworski, A. 30

Tarassow, A. 48

Taylor, A. 91

Taylor, L. 80

Taylor, M. 91

Teglio, A. 83

Tesfatsion, L. 83

Theobald, T. 80

Thirlwall, A. 72, 82, 161

Thomas, R. 99-100

Timmer, M. 130, 163

Tiou Tagba Aliti, G. 78, 82, 83

Tobin, J. 75-76

Toledo, F. 68
Tong, H. 164

Tooke, T. 99

Toporowski, J. 15

Trezzini, A. 40

Tsangarides, C. 54

Tsuda, T. 132

Tytell, I. 7

Valdecantos, S. 78, 82

Van Treeck, T. 41-46, 48, 80, 140

Verdoorn, P. 28, 48, 94

Vergeer, R. 94

Vernengo, M. 92

Verschoor, W. 98

Vianello, F. 35-36, 39

Vicard, V. 116

Vidal, G. 135

Vogel, L. 46, 53-55, 60-64, 66, 91, 114

Walras, L. 30, 75-77

Warnock, F.E. 144

Watson, M. 1, 93

Wei, S.J. 164

White, H.D. 129

Wicksell, K. 21, 30

Wolff, E. 9

Wood, A. 7

Wray, L.R. 79, 99, 159

Yetman, J. 98

Yoo, C.G. 136

You, J.I. 49

Zacharias, A. 9

Zak, G. 103

Zeira, J. 23, 54

Zezza, G. 77-83, 117

Zhao, J. 82

Zlate, A. 145, 165

Zwinkels, R. 98 